\title{
Englisch - die universelle Wissenschaftssprache des 20. Jahrhunderts. Alphonse de Candolle's Prognose von 1873*
}

Huldrych M. Koelbing

\section{Summary}

With his «Histoire des sciences et des savants depuis deux siècles», the Geneva botanist Alphonse de Candolle published, in 1873, the first social history of science. He added to it, among other things, an essay on the necessity of one dominating language suitable to be used by scientists all over the world, and he predicted that, in the $20^{\text {th }}$ century, English would be this language. This, he writes, is sure to happen, above all on account of the previsible demographic evolution. By 1970, the English speaking peoples will greatly outnumber the French and German speaking ones, who are the two other communities prominent in scientific research. By an amiable characterization of the English language, de Candolle makes the inevitable more acceptable to his francophone readers. Finally, he stresses the great responsibility incumbing particularly on the Americans for maintaining their language on a high standard.

\section{Zusammenfassung}

Der vielseitige Genfer Botaniker Alphonse de Candolle publizierte 1873 mit seiner «Histoire des sciences et des savants depuis deux siècles» die erste Sozialgeschichte der Naturwissenschaften. In einem beigefügten Essay betont er die Notwendigkeit einer dominierenden Sprache, in der sich die Wissenschaftler der ganzen Welt verständigen können, und er sagt voraus, dass

* Nikolaus Mani, dem perfekt Polyglotten, gewidmet. Nach einem Vortrag am Symposium zu Ehren von N. Manis 75. Geburtstag.

Prof. Dr. Huldrych M. Koelbing, Gotthardstrasse 65, 8002 Zürich 
dies im 20. Jahrhundert die englische sein wird. Sein Hauptargument ist die voraussehbare demographische Entwicklung: Um 1970 werden die Menschen englischer Muttersprache die beiden andern wissenschaftlich führenden Sprachgruppen, die Deutsch- und Französischsprachigen, an Zahl weit übertreffen. Mit einer liebenswürdigen Charakterisierung der englischen Sprache erleichtert es de Candolle seinen frankophonen Lesern, sich in das Unausweichliche zu finden. Abschliessend betont er die grosse Verantwortung, die insbesondere die Amerikaner dafür tragen werden, das hohe Niveau der englischen Sprache zu erhalten.

\section{Einleitung}

Im Jahre 1873 veröffentlichte der angesehene Genfer Gelehrte Alphonse de Candolle seine «Histoire des sciences et des savants depuis deux siècles» ${ }^{1}$. In denselben Band nahm er eine Reihe von Essays zu wissenschaftlichen Fragen auf. In einem davon erörterte er die Wünschbarkeit, ja die Notwendigkeit einer gemeinsamen Sprache für den Gedankenaustausch unter den Wissenschaftlern der verschiedenen Nationen. Die Analyse der gegebenen Verhältnisse führte ihn zum zwingenden Schluss, dass im kommenden Jahrhundert Englisch diese Rolle spielen werde. Wir haben dabei zu berücksichtigen, dass die französischen Begriffe «science» und «savant» im wesentlichen die Naturwissenschaft und den Naturwissenschaftler bezeichnen; in diesem Bereich lag auch der Hauptberuf des Autors als Botaniker.

\section{Der Autor}

Alphonse Louis Pierre Pyramus de Candolle ${ }^{2}$ gehört einer Dynastie von Genfer Botanikern an, die mit seinem Vater Augustin Pyramus (1778-1841) beginnt. Er selbst kam am 17. Oktober 1806 in Paris zur Welt; hier und in Montpellier verbrachte er seine ersten Lebensjahre. 1816 kehrte die Familie nach Genf zurück, und Alphonse de Candolle lebte fortan in seiner Vaterstadt bis zu seinem Tod am 4. April 1893. Auf Rat seines Vaters studierte er Jurisprudenz, um einen guten Brotberuf zu haben. 1829 promovierte er zum Dr. iur.; aber die Leidenschaft für die Botanik erwies sich als stärker. Mit 25 Jahren wurde er an der Genfer Akademie Dozent für Botanik und später -

1 Alphonse de Candolle, Histoire des sciences et des savants depuis deux siècles, suivie d'autres études sur des sujets scientifiques, en particulier sur la sélection dans l'espèce humaine. Genève, Bâle, Lyon (H. Georg) 1873. 481 p.

2 Siehe P. E. Pilet in: Dict. of Scientific Biography, vol.3, New York 1971, S. 42f.; E. Fueter, Über Bedingungen wissenschaftlicher Leistungen in der europ. Kultur der Neuzeit, in: Gesnerus 8 (1951), 66-84. 
wie sein Vater - Professor dieses Faches. Schon mit 44 Jahren nahm er seinen Rücktritt, um sich ganz seinen persönlichen Studien zu widmen. Seiner Vaterstadt diente Alphonse de Candolle auch in politischen Ämtern; auf seine Initiative ging die Einführung der Briefmarke in Genf im Jahre 1843 die berühmte «Doppel-Genf»-zurück. Als Botaniker machte er sich um die Pflanzengeographie und eine international anerkannte, klare Nomenklatur verdient.

\section{Die Wissenschafts- und Gelehrten-Geschichte}

De Candolle's «Histoire des sciences et des savants» berücksichtigt die Naturwissenschaften und die Mathematik. Sie tut es nicht in der üblichen Weise; de Candolle beschreibt keine Entdeckungen und schildert keine Forscherpersönlichkeiten. Es geht ihm vielmehr um die Geltung der Gelehrten in der Welt und um die gesellschaftlichen Voraussetzungen ihrer herausragenden Leistungen. In dieser Absicht fragt er zunächst danach,

«was die wichtigsten gelehrten Gesellschaften Europas von den Männern gehalten haben, die sich seit zwei Jahrhunderten ausgezeichnet haben» ${ }^{3}$.

Diese gelehrten Sozietäten sind: die Académie des Sciences in Paris, die Royal Society in London und die Akademie der Wissenschaften in Berlin. Aus den Mitglieder-Verzeichnissen sucht de Candolle jeweils die Ausländer heraus, welche diese Akademien in ihre Reihen aufgenommen haben. Auf diese Weise erfasst er das internationale Ansehen der Forscher und Gelehrten.

Weiter fragt er nach der sozialen und örtlichen Herkunft dieser Prominenten und arbeitet so die Faktoren heraus, welche die wissenschaftliche Forschung begünstigen. Schliesslich prüft er die verschiedenen Länder auf das Vorhandensein oder Fehlen dieser (achtzehn) «causes favorables». Dabei nehmen die reformierten Kantone der Schweiz und vor allem die Republik Genf Spitzenränge ein. (Die allgemeine Wehrpflicht, wie sie der schweizerische Bundesstaat seit 1848 kennt, wird allerdings als ein hinderlicher Faktor gewertet, weil der Militärdienst die jungen Männer von ihren Studien abhält!)

Alphonse de Candolle hat also eine Sozialgeschichte der Wissenschaft geschrieben. Dementsprechend hat er in seinem Werk auch die Statistik verwendet - verglichen mit heutigen Usanzen moderat, und vor allem allgemeinverständlich.

3 Op. cit. in Anm. 1, S. 22. 


\section{Die universelle Wissenschaftssprache}

De Candolle's Essay über die Wissenschaftssprache der Zukunft trägt den zwar umständlichen, jedoch präzisen Titel: «Avantages pour les sciences d'une langue dominante, et laquelle des langues modernes sera nécessairement dominante au $\mathrm{XX}^{\mathrm{e}}$ siècle» ${ }^{4}$.

Nach de Candolle ist die Verständigung über alle Grenzen hinweg für Forscher und Gelehrte lebenswichtig, wenn sie auf der Höhe bleiben und die Wissenschaft voranbringen wollen. Sie bereitete wenig Schwierigkeiten, solange alle lateinisch schrieben und diskutierten. Im 17. und 18. Jahrhundert wurde das Latein als gesamteuropäische Gelehrtensprache durch das ihm nahestehende Französisch abgelöst. Im 19. Jahrhundert - für de Candolle ist es die Gegenwart - werden die wichtigsten Arbeiten jedoch in französischer, deutscher oder englischer Sprache publiziert, und jeder Wissenschaftler müsste eigentlich alle drei beherrschen, um sich auf dem Laufenden zu halten. Nach de Candolle gibt es jedoch kaum Leute, denen mehr als zwei Sprachen geläufig sind. Die Wissenschaftler benötigen deshalb für den Austausch ihrer Erkenntnisse und Gedanken dringend eine dominierende Sprache, in der sie sich alle gut verständigen können.

Um in diesem Sinne dominierend zu werden, muss eine Sprache nach de Candolle zwei Grundbedingungen erfüllen. Erstens muss sie germanische wie romanische Elemente enthalten, um den Deutschsprachigen wie den Völkern mit Sprachen lateinischer Herkunft leicht zugänglich zu sein. Zweitens muss sie «von einer beträchtlichen Mehrheit der zivilisierten Menschen» gesprochen werden. Von Vorteil sind zudem eine einfache Grammatik sowie Kürze und Klarheit des Ausdrucks. Und es gibt nur eine Sprache, die in fünfzig bis hundert Jahren - also um 1925-1975 - alle diese Bedingungen erfüllen kann: das Englische. Es ist eine halb germanische, halb lateinische Sprache und verbindet so die jetzt (1873) in der Wissenschaft hauptsächlich gebrauchten Sprachen.

De Candolles entscheidendes Argument ist jedoch quantitativ-demographischer Art:

«Die künftige Ausbreitung der anglo-amerikanischen Sprache ist evident. Sie wird sich durch die Bevölkerungsentwicklung in beiden Hemisphären aufdrängen.» ${ }^{5}$

De Candolle extrapoliert die demographische Entwicklung der nächsten hundert Jahre aus dem Bevölkerungswachstum der letzten Jahrzehnte und kommt zu einem höchst eindrücklichen Ergebnis (siehe Tabelle): 1970 werden auf der Erde 69,5 Millionen Menschen französischer Zunge, 124 Mil-

4 Op. cit., S. 292-307.

5 Op. cit., S. 296. 
Tabelle. Sprachgruppen in der Welt (in Millionen)

\begin{tabular}{lccl}
\hline & Englisch & Deutsch & Französisch \\
\hline nach A. de Candolle (1873) & & & \\
1870: & 77 & 62 & 40,5 \\
1970: & 860 & 124 & 69,5 \\
tatsächlich & & & \\
1990: & 350 & 95 & 70 \\
\hline
\end{tabular}

lionen Deutschsprachige und 860 Millionen Englischsprechende leben! Inzwischen hat sich gezeigt, dass die Anglophonen sich nicht ganz so gewaltig vermehrten, wie de Candolle annahm - 1990 waren es rund 350 Millionen -; aber in den grossen Zügen sah er die Entwicklung richtig voraus.

Man kann, wenn man will, bemängeln, dass der weitblickende Genfer nicht nur die fernen Chinesen, sondern auch die heutige Weltsprache Spanisch vernachlässigt hat. Doch er hielt sich an diejenigen Sprachgruppen, die zu seiner Zeit in der Forschung führend waren und von denen er annahm, dass sie es auch im nächsten Jahrhundert bleiben würden. Und seine Voraussage hat sich vollkommen bestätigt: Englisch ist im 20. Jahrhundert die auf der ganzen Welt dominierende, die universelle Sprache der Wissenschaft geworden. Mehr noch: Auch im täglichen Leben, in Handel und Verkehr dominiert heute Englisch rund um die Erde.

\section{Die englischsprechenden Völker und ihre Sprache}

De Candolle gibt sich mit seinen Grundregeln und Zahlen nicht zufrieden, sondern geht noch etwas näher auf die englische Sprache und ihre Sprecher ein. Dass in Nordamerika und Australien Literatur und Wissenschaft noch nicht im gleichen Ausmass gepflegt werden wie in Europa, räumt er durchaus ein; Landwirtschaft, Industrie und Handel werden dort vielleicht noch lange die aktivsten Kräfte absorbieren. Aber eine so grosse Masse intelligenter und gebildeter Menschen ist auf jeden Fall ein Faktor von entscheidendem Gewicht in der Welt. Zudem:

«Diese neuen Völker englischen Ursprungs sind mit Deutschen durchmischt, die bezüglich der intellektuellen Neigungen die Irländer kompensieren. Sie sind allgemein lernbegierig und brennen darauf, Entdeckungen anzuwenden.»

Und sie lesen viel. Damit liegt für Bücher in englischer Sprache, auch für Übersetzungen, ein verheissungsvolles Feld weit offen. Wohl etwas allzu optimistisch schreibt de Candolle:

6 Op. cit., S. 298. 
«Wir in Europa wissen, wie schwierig es ist, seriöse Bücher zu publizieren. Aber eröffnen Sie dem Buchhandel einen immensen Markt und die speziellsten Werke werden sich verkaufen lassen.» ${ }^{7}$

Die englische Sprache hat einen einzigen kapitalen Nachteil: ihre völlig regellose, absurde Orthographie ${ }^{8}$ ! Auf der andern Seite besitzt sie jedoch einen sozusagen familiären Vorzug, der ihr Erlernen begünstigt:

«Ein junger Mann, der seinen Sprachlehrer recht pedantisch und seine Grammatik höchst langweilig findet, hat nicht denselben Eindruck, wenn seine Mutter, seine Schwester oder eine Freundin seiner Schwester sich in einer fremden Sprache an ihn wendet. Oft wird das auf englisch sein, mit vortrefflichem Grund. Keine andere Sprache ist so reich an Werken, die - vollkommen schicklich geschrieben - thematisch die Frauen ansprechen: Bücher über Religion und Erziehung, Romane, Memoiren, Poesie etc.

Die künftige Vorherrschaft der Sprache der Engländer, Australier und Amerikaner scheint mir also gesichert...»9

Daraus erwächst diesen Völkern, vor allem den Amerikanern, allerdings eine grosse Verantwortung, nämlich die Aufgabe, die englische Sprache auf ihrem hohen Niveau zu erhalten und so weiterzugeben ${ }^{10}$. Sind sie diesem Auftrag gerecht geworden? Gewiss nicht in dem von de Candolle gewünschten Mass. Selbstverständlich gibt es auch heute noch, im Vereinigten Königreich und in Übersee, wissenschaftliche Autoren, die ein schönes und reiches, nuanciertes Englisch schreiben und darin Fakten und Gedanken präzis und fasslich wiedergeben. Und es gibt Redaktoren, die darauf halten. Doch daneben macht sich ein verarmtes Allerwelts-Englisch breit, in dem sich banale Ausdrücke und Wendungen häufen und Sachverhalte nur approximativ getroffen werden. Qualität und Präzison des sprachlichen Ausdruckes scheint manchen unwichtig - wenn nur die reichlich mitgeteilten Zahlen einigermassen stimmen! Diese sprachliche Unbeholfenheit ist natürlich häufiger und grösser bei denjenigen, für die Englisch eine Fremdsprache ist, deren sie sich notgedrungen bedienen müssen, um überhaupt beachtet zu werden.

Doch de Candolles Mahnung gilt im Grunde gewiss nicht nur für Amerikaner und für Naturwissenschaftler. Wir alle sind für die Sprache verantwortlich, vor allem für unsere eigene. Es kommt nicht nur darauf an, was wir als Wissenschaftler zu sagen haben, sondern auch, wie wir es sagen und schreiben.

7 Op. cit., S. 299.

8 Op. cit., S. 301.

9 Op. cit., S. 303f.

10 Op. cit., S. 304-306.

13 Gesnerus 